\title{
The importance of redundancy of functional ovarian reserve when investigating potential genetic effects on ovarian function
}

\author{
David H. Barad ${ }^{1,2}$ • Vitaly A. Kushnir ${ }^{1,3}$ • Norbert Gleicher ${ }^{1,2,4}$
}

Received: 11 May 2016 / Accepted: 22 June 2016 / Published online: 16 July 2016

(C) Springer Science+Business Media New York 2016

Until recently, the Fragile X mental retardation 1 (FMRl) gene, located at Xq27.3, received only limited attention in reproductive medicine since known associated medical conditions were mostly neuro-psychiatric. Fragile $\mathrm{X}$ syndrome (FXS), due to FMR1 full mutations $\left(\mathrm{CGG}_{n}>200\right)$, is the most common form of familial mental retardation. In middle aged males, FMRI's premutation range, at approximately $\mathrm{CGG}_{n=55-200}$, is characterized by phenotypic expression of a neurodegenerative disease known as the Fragile Xassociated tremor/ataxia syndrome. In females, the premutation range $\mathrm{CGG}_{n=55-200}$ is associated with an increased risk of primary ovarian insufficiency (POI). Women with premutation range $\mathrm{CGG}_{n=55-200}$ have a significant onegenerational risk that their offspring may demonstrate full mutation range $\mathrm{CGG}$ expansions and, therefore, FXS. For this reason, pre-conception screening for maternal premutations is now commonly offered. The so-called intermediate (or "gray" zone), between approximately $\mathrm{CGG}_{n=45-54}$, carries only minimal risk for one-generational expansion to FXS, while the classical normal (or "common") range of $\mathrm{CGG}_{n<45}$ carries no such risk at all [1].

Capsule Studies, especially cross-sectional studies, of genes that affect functional ovarian reserve (FOR) should only be performed in women who have begun to lose redundant FOR.

David H. Barad

dbarad@theCHR.com

1 The Center for Human Reproduction (CHR), New York, NY, USA

2 The Foundation for Reproductive Medicine, New York, NY, USA

3 Department of Obstetrics and Gynecology, Wake Forest School of Medicine School of Medicine, Winston Salem, NC, USA

4 Stem Cell and Molecular Embryology Laboratory, The Rockefeller University, New York, NY, USA

\section{Background}

More than 7 years ago, the observation that the FMR1 gene was associated with POI raised suspicion that it may have broader clinical associations with ovarian function than was appreciated at the time [2]. In the general population FMR1 $\mathrm{CGG}_{\mathrm{n}}$ demonstrates a large peak around $\mathrm{CGG}_{n=29-30}$ [3]. We inferred that this peak represented a range of normal FMR1 function. We further reasoned that if the FMR1 gene has a role in normal ovarian reproductive function then FMR1 mutations significantly different from the population median might also influence ovarian reserve. In a series of investigations, we then defined a new normal "ovarian" range for the FMR1 gene at $\mathrm{CGG}_{n=26-34}$, which included the distribution peak at $\mathrm{CGG}_{n=29-30}$ [2].

We observed variations in serum anti-Müllerian hormone $(\mathrm{AMH})$, an indicator of functional ovarian reserve (FOR), that were associated with FMR1 CGG below and above $\mathrm{CGG}_{n=26-34}$. We were surprised to observe that the "effect" of these FMR1 mutations was different in different age groups, which suggested varying patterns of loss of FOR associated with age. Until then, research on the FMR1 gene had mostly concentrated on expansion ranges of $\mathrm{CGG}_{n>45}$. In our studies, surprisingly, low alleles $\left(\mathrm{CGG}_{n<26}\right)$ demonstrated the most profound reproductive effects [2].

Since then, a number of studies have attempted to further clarify effects of CGG repeats within the classical normal range. We will address three of these recent studies [4-6] that each investigated young healthy fertility patients and found only limited evidence of effect of FMR1, within the classical normal range, on indicators of ovarian reserve. We maintain that, when studying a genetic condition that may predispose to age related loss of FOR, the conclusions of a study will have little meaning unless a population at risk was studied and that young healthy fertility patients are low risk for low FOR. 


\section{Importance of redundancy in FOR}

We recently noted in this journal that patient selection is important in interpreting outcome data in clinical reproductive medicine [7]. Young women, even those who may have a genetic predisposition for a future loss of FOR, have normal ovarian function because they benefit from a redundancy of developing follicles, which we define as redundant functional ovarian reserve (redundant FOR). When studying the effect of genetic conditions on ovarian function, patient selection is of the greatest importance. The extraordinary level of redundant FOR that exists among normal young women can mask an effect when observed in a cross-sectional study because of the need for age/time-dependent study of ovarian function.

Here is a good FMR1 gene-related example of why patient selection is so important: FOR of young oocyte donors does not vary (based on AMH levels) unless a young woman is unlucky enough to have homozygosity for two low FMR1 alleles. The much more frequent heterozygous (single) low FMR1 genotype (present in ca. 20-25\% of women) has not yet effected FOR in the very young woman. Even so, after only 4 years of follow-up of young oocyte donors with one low FMR 1 allele, we were able to see that AMH levels significantly fell compared to those of young oocyte donors without low FMR1 alleles [8].

Thus, young women with heterozygous low FMR1 alleles demonstrate, at first, less severe, and later clinically overt effects on ovarian reserve; and, when homozygous, more severe, and earlier emergence of a clinical phenotype. Yet, although young oocyte donors with low FMR1 alleles may demonstrate lower FOR (as measured by $\mathrm{AMH}$ ) than donors who lack such alleles, this does not mean they have clinically impaired fertility. These donors still produce similar pregnancy and live birth rates in recipients as egg donors without low FMR1 alleles [8], though the latter, indeed, may produce lower cumulative pregnancy rates because their mildly lower FOR produces fewer eggs and embryos [9]. Therefore, it is important to recognize that redundancy in female FOR may hide the effect of low FMRI alleles and will cause clinical infertility only once FOR has lost its protective redundancy. Such a masking effect may also be present for other genetic conditions that could impact the patterns of age related loss of ovarian function.

An understanding that redundancy of FOR can affect the clinical expression of a predisposition to premature ovarian aging/occult premature ovarian insufficiency (POA/oPOI) is, therefore, critical in interpreting FMR1 studies. FMR1 mutations neither diagnose low FOR nor female infertility. Low FMR1 mutations denote risk toward POA/oPOI and, therefore, predict the possibility that a woman will have low FOR and infertility at some point in the future, once her FOR redundancy has disappeared.
Therefore, if FMRl studies are performed in young women who still have a high degree of redundant FOR, significant expression of clinical ovarian function differences between FMR1 genotypes will not be apparent. Only advancing female age and/or POA/oPOI, resulting in loss of redundancy of FOR, will make FMR1 effects visible. The type of patient who is investigated in FMR1 studies of ovarian reserve is, therefore, of crucial importance.

\section{Recent studies}

Banks et al. recently reported an analysis of over 3000 infertile women undergoing in vitro fertilization (IVF), so far the largest FMR1 study in the reproductive medicine literature [4]. They demonstrated associations of some FMR1 mutations with oocyte yields and other markers of FOR and thus, at least partially, confirmed our previous observations. Moreover, the associations they observed were with $\mathrm{CGG}_{\mathrm{n}}$ mutations in what had been considered the normal ("common") and intermediate ("gray zone") triple CGG range, both ranges widely considered clinically unimportant before our initial FMR1 publications [2].

Banks et al. were able to confirm in their study that the socalled low FMR1 mutations $\left(\mathrm{CGG}_{n<26}\right)$ were associated with evidence of decreased FOR [2]. They reported that the observed associations were significantly weaker than previously reported in our studies. However, they studied very different patient populations than we did. Banks et al. reported that median ages of their study population were between 34 and 36 years, median FSH was from 6.8 to $7.9 \mathrm{mIU} / \mathrm{mL}$, and median AMH ranged from 1.8 to $2.3 \mathrm{ng} / \mathrm{mL}$ [4]. Their IVF patients, thus, were very favorably selected and to a significant degree excluded women with low FOR. In contrast, the typical patient selected in one of our recent studies was 39.7 years old, had a mean FSH of $11.2 \mathrm{mIU} / \mathrm{mL}$, and mean AMH of only $1.5 \mathrm{ng} / \mathrm{mL}$ [10]. Thus, Bank's patient population provided less power to detect a possible association with impaired FOR and for this reason the observed association was weaker than previously reported in a more at-risk population.

Banks et al. also reported that the weak associations with oocyte yields and other markers of ovarian reserve further attenuated after adjustments for patient age, $\mathrm{AMH}$, antral follicle count, and FSH [4]. Assuming that FMR1 exerts genetic effects on the ovary, its potential effects on ovarian function are only one part of a chain of events leading to low FOR and consequently low antral follicle count, high FSH, and low AMH. It, therefore, makes little sense to adjust for indicators of FOR that are further downstream. This would be like adjusting for the effect of a dam, based on the downstream water flow or like claiming that a 5 -ft tall center in basketball is, after adjustment for height, equally effective as a seven footer. Such adjustments make no sense since the second 
factor depends upon the first and the adjustments simply cancel each other out.

Recently, Morin et al. reported that $\mathrm{CGG}_{\mathrm{n}}$ is not predictive of ovarian response in IVF cycles [5]. However, once again, the patient population investigated was largely comprised of good prognosis patients. The patient's mean age was 34.9 years with a mean of 11.1 mature oocytes per retrieval which suggests generally good FOR. One, therefore, could conclude that had Morin et al. studied their hypothesis in a less favorably selected patient population they may have found a different result.

Finally, in this issue of JARG, Benadiva et al., in a study of 603 women, found a higher proportion of age adjusted decreased ovarian reserve among patients with homozygous low FMR1 alleles, although they did not detect any associations between other FMR1 mutations and FOR [6]. Interestingly, in earlier versions of this analysis, the authors were unable to see this effect. Only after the review process when the reviewer asked for the analysis to be age stratified, was the effect of the homozygous low alleles detectable. Furthermore, with mean age of 33.5 years, mean FSH $6.6 \mathrm{mIU} / \mathrm{mL}$, and mean AMH $2.2 \mathrm{ng} / \mathrm{mL}$, their study population was even younger and more favorably selected than the prior two studies reviewed $[4,5]$.

\section{Conclusions}

The three studies discussed provide support for our argument that genetic control of ovarian aging can only become clinically visible once FOR redundancies are exhausted.

Banks et al., despite their large study cohort, only were able to demonstrate marginal associations with the FMRI gene [4]. Morin et al. were unable to demonstrate any association of FMR1 mutations and ovarian response, which is not surprising given that their study cohort had evidence of even more favorable ovarian reserve [5]. Finally, in another highly selected group [6], were only able to demonstrate an effect of FMR1 among subjects who were homozygous low/low [9]. This is exactly what one would expect in this group of patients with mostly favorable age related FOR.

One lesson we can derive from the three recently published FMR1 studies [4-6] is that, in order to achieve interpretable results, studies of genetic effects on FOR should be performed on women who no longer exhibit significant redundancies in FOR. If studies are performed in young, good prognosis patients, then cross-sectional studies will have less power to detect an effect. Such studies, as we demonstrated in young oocyte donors [8], have to be prospective and follow young women longitudinally over a number of years.
In this commentary, we have attempted to explain the importance of FOR redundancy and patient selection in attempting to demonstrate associations between genetic effects and age related ovarian function. Though we cannot concur with their conclusions, we are grateful to Banks et al. [4] and Morin et al. [5] and the authors of the manuscript in this issue [6] for their efforts because, in their respective degrees of IVF patient selection, they allowed us to demonstrate our arguments in practice rather than only in theory.

We hope to have clarified why clinical studies of the FMR1 gene have yielded such divergent results. Animal studies have increasingly confirmed the likely importance of the FMR1 gene in ovarian physiology. Recently FMR1 gene product was demonstrated present at all stages of folliculogenesis in the rat $[11,12]$ and in a mouse model of ovarian aging [13]. Given the accumulating animal data together with the effects seen in cross-sectional studies, it may be time for longitudinal study of FMR1 effects in women in their early 20 s for a period of approximately 10 years. Such a study would require federal funding and, likely, multicenter collaboration but would offer the quickest and most reliable opportunity to establish clinical significance of individual FMR1 mutations. We also need further investigation into the role of the FMR1 gene on animal and human ovarian physiology at the molecular level.

Finally, considering all published literature on effects of the FMR1 gene on ovarian aging, it appears reasonable to suspect that low FMR1 alleles in young women may represent a significant risk for the later development of POA/oPOI. In addition, women with evidence of low FOR experience a 30-40\% lower chance of clinical pregnancy in IVF compared to those women with low FOR who lack low FMR1 alleles [2, 10, 14]. The FMR1 gene, thus, appears to affect reproductive success to a very significant degree and deserves further exploration in appropriately selected patient populations.

Acknowledgments This work was supported by intramural funds from The Center for Human Reproduction and grants from The Foundation for Reproductive Medicine.

\section{Compliance with ethical standards}

Competing interests All authors have read the journal's policy on disclosure of potential conflicts of interest and have the following disclosures to make: N.G. and D.H.B. are co-inventors on a number of pending and already awarded US patents claiming therapeutic benefits from androgen supplementation in women with low functional ovarian reserve (LFOR) and relating to the FMR1 gene in a diagnostic function in female fertility. Both receive royalties from Fertility Nutraceuticals, LLC, in which N.G. also holds shares. N.G., D.H.B., and V.A.K. also are coinventors on a pending AMH-related patent application. They report no other potential conflicts with here reported manuscript. All other authors report no potential conflicts with here reported manuscript. 


\section{References}

1. Wittenberger MD, Hagerman RJ, Sherman SL, McConkie-Rosell A, Welt CK, Rebar RW, et al. The FMR1 premutation and reproduction. Fertil Steril. 2007;87(3):456-65. doi:10.1016/j.fertnstert.2006.09.004.

2. Gleicher N, Kushnir VA, Weghofer A, Barad DH. How the FMR1 gene became relevant to female fertility and reproductive medicine. Front Genet. 2014;5:284. doi:10.3389/fgene.2014.00284.

3. Fu YH, Kuhl DP, Pizzuti A, Pieretti M, Sutcliffe JS, Richards S, et al. Variation of the CGG repeat at the Fragile X site results in genetic instability: resolution of the Sherman paradox. Cell. 1991;67(6):1047-58.

4. Banks N, Patounakis G, Devine K, DeCherney AH, Widra E, Levens ED, et al. Is FMR1 CGG repeat length a predictor of in vitro fertilization stimulation response or outcome? Fertil Steril. 2016;105(6):1537-46 e8. doi:10.1016/j.fertnstert.2016.02.011.

5. Morin SJ, Tiegs AW, Franasiak JM, Juneau CR, Hong KH, Werner $\mathrm{MD}$, et al. FMR1 gene $\mathrm{CGG}$ repeat variation within the normal range is not predictive of ovarian response in IVF cycles. Reprod Biomed Online. 2016;32(5):496-502. doi:10.1016/j.rbmo.2016.02.009.

6. Maslow B-SL, Davis S, Engmann L, Nulsen JC, Benadiva CA. Correlation of normal-range FMR1 repeat length or genotypes and reproductive parameters. J Assist Reprod Genet. 2016. doi:10.1007/s10815-016-0732-2.

7. Gleicher N, Kushnir VA, Barad DH. The impact of patient preselection on reported IVF outcomes. J Assist Reprod Genet. 2016. doi:10.1007/s10815-016-0673-9.
8. Gleicher N, Yu Y, Himaya E, Barad DH, Weghofer A, Wu YG, et al. Early decline in functional ovarian reserve in young women with low $(\mathrm{CGGn}<26)$ FMR1 gene alleles. Transl Res. 2015;166(5):502-7 e1-2. doi:10.1016/j.trsl.2015.06.014.

9. Gleicher N, Weghofer A, Barad DH. Can egg donor selection be improved?-A pilot study. Reprod Biol Endocrinol. 2010;8:76. doi:10.1186/1477-7827-8-76.

10. Kushnir VA, Yu Y, Barad DH, Weghofer A, Himaya E, Lee HJ, et al. Utilizing FMR1 gene mutations as predictors of treatment success in human in vitro fertilization. PLoS One. 2014;9(7): e102274. doi:10.1371/journal.pone.0102274.

11. Ferder I, Parborell F, Sundblad V, Chiauzzi V, Gomez K, Charreau $\mathrm{EH}$, et al. Expression of Fragile $\mathrm{X}$ mental retardation protein and Fmr1 mRNA during folliculogenesis in the rat. Reprod. 2013;145(4):335-43. doi:10.1530/REP-12-0305.

12. Takahashi N, Tarumi W, Itoh MT, Ishizuka B. The stage- and cell type-specific localization of Fragile $\mathrm{X}$ mental retardation protein in rat ovaries. Reprod Sci. 2015;22(12):1524-9. doi:10.1177 /1933719115589416.

13. Hoffman GE, Le WW, Entezam A, Otsuka N, Tong ZB, Nelson L, et al. Ovarian abnormalities in a mouse model of Fragile $X$ primary ovarian insufficiency. J Histochem Cytochem. 2012;60(6):439-56. doi:10.1369/0022155412441002.

14. Gleicher N, Weghofer A, Lee IH, Barad DH. Association of FMR1 genotypes with in vitro fertilization (IVF) outcomes based on ethnicity/race. PLoS One. 2011;6(4):e18781. doi:10.1371/journal. pone. 0018781 . 